\title{
Energy efficient CF-OFDM scheme with single IFFT modulator for broadband wireless devices
}

\begin{abstract}
In many low-cost mobile device applications, the drawback of high crest factor (CF) may outweigh all the potential benefits of broadband communication systems. Orthogonal frequency division multiplexing (OFDM) is considered to be one of the important multicarrier standards in broadband communication systems. However, high $\mathrm{CF}$ is the major problem of OFDM, which may have a deleterious effect on battery lifetime on broadband wireless devices. Therefore, applying a low-complexity high-efficiency technique in recent communications standard will significantly reduce the complexity of those systems and bring down the cost of the system. In this paper, a novel scrambling CF reduction scheme to reduce the CF in OFDM systems is proposed. In this scheme, two re-ordering methods are proposed. Then, a new optimization scheme is introduced in which only a single two-phase sequence need to be applied. Unlike the conventional-partial transmit sequence (C-PTS) scheme which needs Q-IFFT modulators, the proposed scheme requires only a single IFFT modulator. This feature significantly reduces processing time and less computation that leads to reduced complexity. Simulation results demonstrate that the proposed scheme can effectively reduce the complexity compared with the conventional and latest $\mathrm{CF}$ reduction scheme and yields good CF performance.
\end{abstract}

Keyword: OFDM; CF; PAPR; Re-ordering method; PTS 